\title{
The Optimal Scheme of Space Debris Mitigation
}

\author{
Yue Song ${ }^{1, a}$ \\ ${ }^{1}$ School of North China Electric Power University, Baoding 071000, China. \\ a15733229653@163.com
}

Keywords: Space Debris, Economic Indicator, Commercial Opportunity, The Optimal Proportion

\begin{abstract}
In this paper, a time-dependent model is established to determine the optimal scheme which can bring economically attractive opportunity to a private firm. yield was taken into account in this model (yield is a variable varies from time). We build a time-dependent model to balance the costs and benefits, verifying the private firm is able to profit in the long term. Implementing simulation, we determined the best scheme is combination removal; meanwhile, we obtain the best proportion of the number of space debris using laser removal to the number of space debris using satellite removal.
\end{abstract}

\section{Introduction}

Since the number of satellites in earth orbit is steadily increasing, space debris, if left unchecked, will eventually pose a serious hazard to near-Earth space activities, so effective measures should be taken to mitigate it. The major concern about debris is that it might hit the operational spacecraft or a larger object such as the International .

Space Station, with a whole variety of detrimental consequences. Space debris has been increased so quickly that the population of such orbital debris has risen to the point where the likelihood of collision can't be ignored. It is urgent to handle orbital debris issues from different ways since orbital debris is a serious threat for operational spacecraft, especially for manned spacecraft.

There are lots of schemes to remove space debris and two of them are explored in this paper. They are laser removal and satellite removal. Based on this, we designed three schemes for private firm: laser removal, satellite removal and combination scheme.

To solve the problem, yield was taken into account (yield is a variable varies from time). We build a cost-benefit model to determine if the private firm is able to profit in the long term.

\section{The Optimal Scheme Selecting Model}

\subsection{Redistribution of Weight}

In order to discuss whether an economically attractive opportunity exists or not, we focus on the cost and benefits of removal methods and avoiding collisions.

Here we redistribute the weight of four economic indicators.

We established a new judgment matrix $J^{\prime}$ as follows:

$$
J^{\prime}=\left[\begin{array}{cccc}
1 & \frac{1}{5} & \frac{1}{5} & 3 \\
5 & 1 & 3 & 7 \\
3 & \frac{1}{3} & 1 & 5 \\
\frac{1}{3} & \frac{1}{7} & \frac{1}{5} & 1
\end{array}\right]
$$

So the new sort weight of four economic indicators is:

$$
\left[\begin{array}{llll}
w_{R}{ }^{\prime} & w_{C}{ }^{\prime} & w_{B}{ }^{\prime} & w_{I}{ }^{\prime}
\end{array}\right]=\left[\begin{array}{llll}
0.0654 & 0.4972 & 0.2926 & 0.1449
\end{array}\right]
$$




\subsection{Cost Analysis}

Based on the above model, it is apparently that small piece of space debris is the majority in total. Consider comprehensively, we mainly discuss the situation when the assemble density is high. Meanwhile, we take the situation of avoiding space debris only into account, considering it as a space debris removal scheme.

\subsubsection{Numerical Calculation}

With the development of technology, the cost will be falling over time. We assume that initial average decline rate $\sigma$ will change over time. The decline rate rt will gradually reduce and after a period of time it can be expressed as,

$$
r_{t}=q \sigma^{t}
$$

Where $\mathrm{q}$ is the coefficient of ${ }^{r_{t}}$, then the cost is

$$
C_{t}=C_{0}\left(1-r_{t}\right)
$$

Where C0 is the initial cost and plugging $r_{t}$ into the Eq.(21), we obtain Eq.(22) as follows,

$$
C_{t}=C_{0}\left(1-q \sigma^{t}\right)
$$

Then according to Eq.3, we can get the costs of four kinds of removal schemes which shown in Tab.1

Tab.1 Cost of all kinds of considering alternatives

\begin{tabular}{cc}
\hline Alternative & Cost \\
& $\%$ \\
\hline Laser removal & $0.015 \mathrm{n}+1.75$ \\
Satellites removal & $0.020 \mathrm{n}+0.75$ \\
Combination scheme & $0.015 \mathrm{n} 1+0.020 \mathrm{n} 2+0.9$ \\
Avoiding only scheme & $0.012 \mathrm{n}+1.16$ \\
\hline
\end{tabular}

Where $n$ is the quantity of space debris, $n 1$ is the number of debris in the first removal method, $n 2$ is the number of debris in the second removal method.

2.2.2 Consequence Analysis

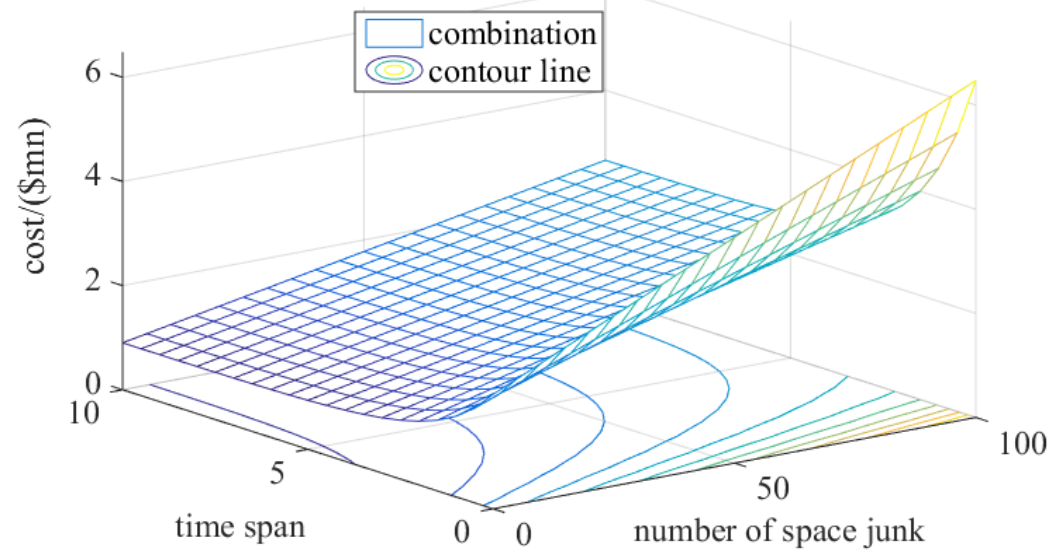

Fig.1 The cost of combination scheme relate to time and number of junk

As is indicated in Fig.1, taking combination situation for example, the cost is closely connected with time and the number of space debris. The increasing of space debris will cause the growth of costs at a time. In other aspect, when dealing with fixed number debris, the cost increases as the time goes.

\subsection{Benefits Analysis}

2.3.1 Numerical Calculation 
With the development of space technology, the value of satellites or aircrafts will increase by years. The benefits of a private firm will increase in the same time.

We assume the initial increasing rate of benefits is $\omega$, after a period, the increasing rate $\varphi$ will be

$$
\varphi=p \omega^{t}
$$

Where $\mathrm{p}$ is the coefficient of $\omega^{t}$, then the benefits Bt will be

$$
B_{t}=B_{0}(1+\varphi)
$$

Where B0 is the initial benefits, putting $\varphi$ into Eq.24, we gain

$$
B_{t}=B_{0}\left(1+p \omega^{t}\right)
$$

\subsubsection{Consequence Analysis}

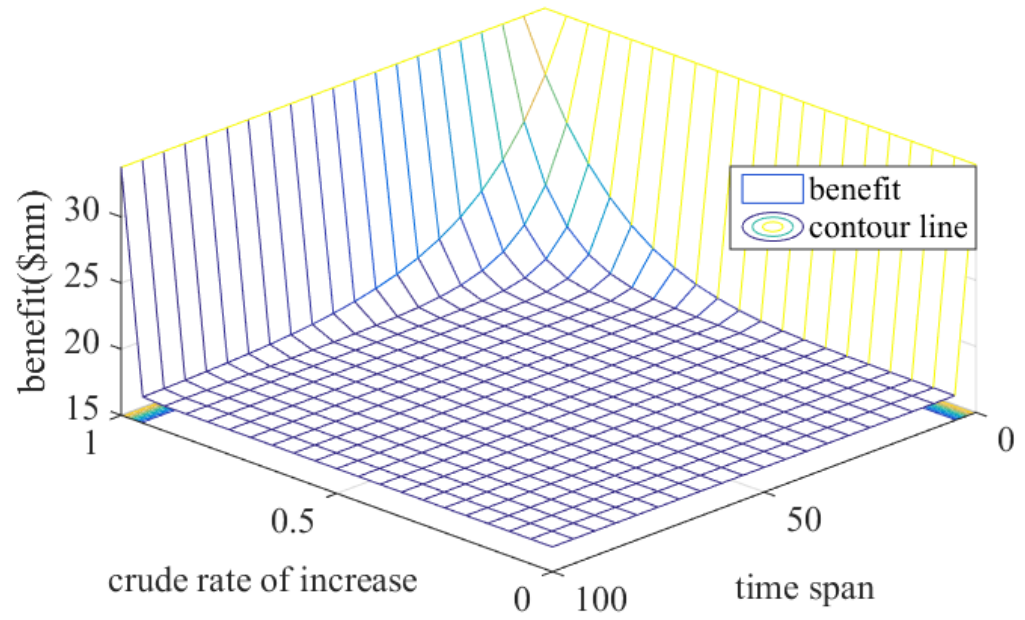

Fig. 2 the benefits relate to time and crude rate of increase fit to all schemes

As is shown in the Fig.2,

1. The benefits grow with the increasing of the initial growth rate at a time.

2. As time increasing, the benefit will decrease in a certain initial increasing rate.

3. After a long time, the benefit almost won't change.

\subsection{The Optimal scheme for Commercial Opportunity}

In this model, the estimated values of economic indicators are shown in the Tab.2, when using avoiding only scheme, the interference degree is highest, which is equal to 1 . In order to analyze and

\begin{tabular}{|c|c|c|c|c|c|}
\hline \multicolumn{2}{|c|}{$\begin{array}{l}\text { Range of collision } \\
\text { probabilities }\end{array}$} & \multirow{2}{*}{ 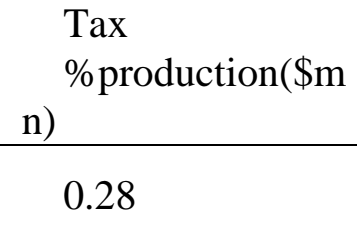 } & \multirow{2}{*}{$\begin{array}{l}\begin{array}{l}\text { Cost } \\
\%\end{array} \\
(0.015 n+1.05)\end{array}$} & \multirow{2}{*}{$\begin{array}{l}\text { Benefi } \\
\text { ts } \\
16.87\end{array}$} & \multirow{2}{*}{$\begin{array}{c}\text { ce }^{\text {interferen }} \\
0.25\end{array}$} \\
\hline \multirow{4}{*}{0.003} & Energy laser & & & & \\
\hline & satellites & 0.20 & $(0.020 n+0.75)$ & 16.87 & 0.3 \\
\hline & $\begin{array}{l}\text { Combination } \\
\text { of energy laser } \\
\text { and satellites }\end{array}$ & 0.24 & $\begin{array}{l}\quad(0.015 n 1+0.020 n \\
2+0.9)\end{array}$ & $5^{16.87}$ & 0.5 \\
\hline & $\begin{array}{l}\text { Avoiding } \\
\text { collisions }\end{array}$ & 0.31 & $(0.012 n+1.16)$ & $5^{16.87}$ & 1 \\
\hline
\end{tabular}
choose the optimal scheme, a figure is given in Fig.3 (the number of space debris $n$ is equal to 500).

Tab. 2 The estimation values of economic indicators 


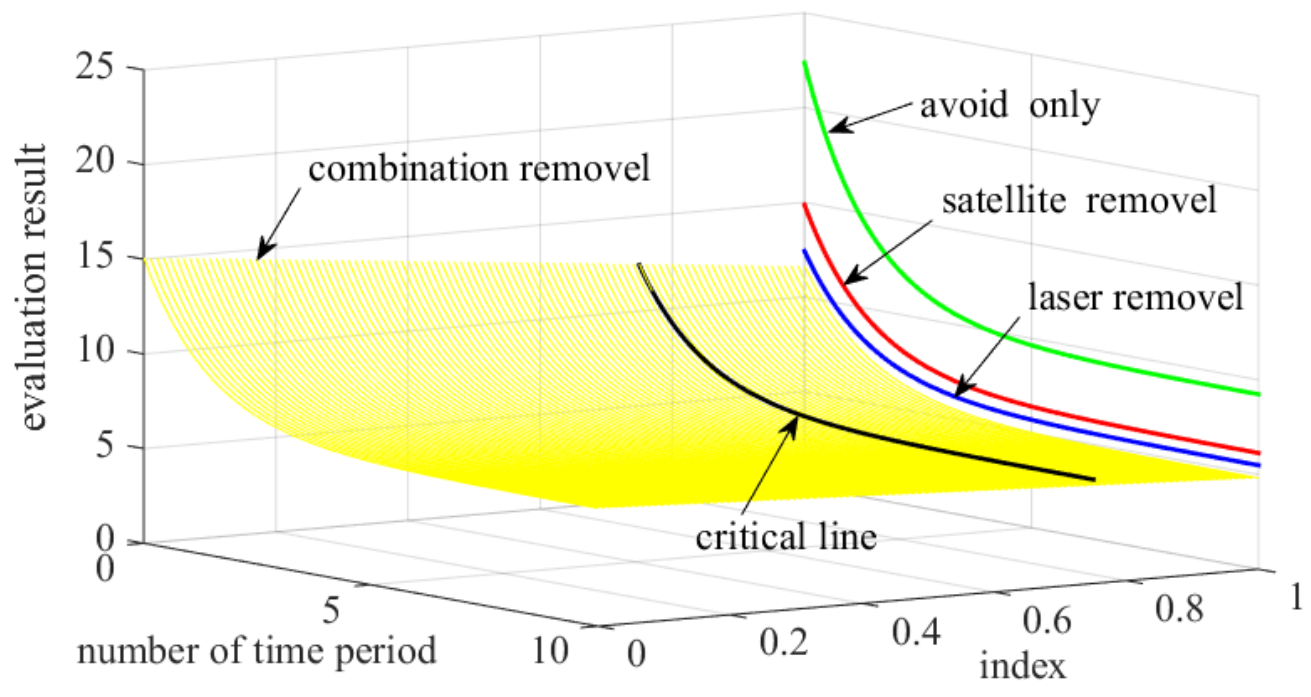

Fig.3 evaluation result

As is indicated in Fig.3, the consequences shows two kinds of extreme cases in which the effect of avoid-only method is poor, and collisions also bring enormous losses. For example, the loss of Orion 3 satellite in 1999 cost insurers \$265 million dollars and added 645 million dollars in revenue losses. so we can draw the conclusion that there exists good market prospect in this domain.

According to Fig.3, it is apparently that the combination scheme is optimal and the optimal proportion $\mathrm{b}$ is around $74 \%$. Though the higher ratio may bring more profit, considering the necessary of long-term demand, the best proportion is $74 \%$.

\section{Summary}

In this paper, we discuss whether an economically attractive opportunity exists. Comparing three debris removal methods with avoiding collision in aggregative indicators, we consider the commercial opportunity exists, and the combination case is optimal. The primary outcome indicates as: two kinds of extreme cases, avoid only or collisions, are not the selectable schemes, while the combinational scheme is optimal. The debris's quantity using laser removal method account for about $74 \%$ of total quantity with combination scheme and it is optimal in combination method. Though the higher ratio brings more profit, it had better satisfied long-term demand for various situations, so the optimal proportion is about $74 \%$.

\section{References}

[1] J.C. Dolado-Perez, Carmen Pardini, Luciano Anselmo, Review of uncertainty sources affecting the long-term predictions of space debris evolutionary models, Acta Astronautica, 2015, PP 51-65.

[2] http://dx.doi.org/10.1016/j.actaastro.2015.03.033.

[3] Minghe Shan, Jian Guo, Eberhard Gill, Review and comparison of active space debris capturing and removal methods, Progress in Aerospace Sciences, 2016, PP 18-32.

[4] Minghe Shan, Jian Guo, Eberhard Gill, Review and comparison of active space debris capturing and removal methods, Progress in Aerospace Sciences, 2016, PP 18-32

[5] Niels van der Pas, Joao Lousada, Claudia Terhes, Marc Bernabeu, Waldemar Bauer, Target selection and comparison of mission design for space debris removal by DLR's advanced study group, Acta Astronautica, 2014, PP 241-248. 